Cahiers $d u$ MONDE RUSSE

\section{Cahiers du monde russe}

Russie - Empire russe - Union soviétique et États indépendants

$43 / 4 \mid 2002$

Intellectuels et intelligentsia

\title{
N. N. Smirnov, ed., Rossija i pervaja mirovaja voina
}

\section{Alessandro Stanziani}

\section{OpenEdition \\ Journals}

Édition électronique

URL : https://journals.openedition.org/monderusse/4020

DOI : 10.4000/monderusse. 4020

ISSN : $1777-5388$

Éditeur

Éditions de l'EHESS

Édition imprimée

Date de publication : 30 décembre 2002

Pagination : 705-708

ISBN : 2-7132-1796-2

ISSN : $1252-6576$

\section{Référence électronique}

Alessandro Stanziani, « N. N. Smirnov, ed., Rossija i pervaja mirovaja voina », Cahiers du monde russe [En ligne], 43/4 | 2002, mis en ligne le 17 juin 2009, consulté le 03 septembre 2022. URL : http:// journals.openedition.org/monderusse/4020 ; DOI : https://doi.org/10.4000/monderusse.4020

Ce document a été généré automatiquement le 3 septembre 2022

Tous droits réservés 


\title{
N. N. Smirnov, ed., Rossija i pervaja mirovaja voina
}

\author{
Alessandro Stanziani
}

\section{RÉFÉRENCE}

N. N. SMIRNOV, ed., Rossija i pervaja mirovaja voina (La Russie et la Première Guerre mondiale). Saint-Pétersbourg, Dmitrij Bulanin, 1999, 563 p.

1 Si la Première Guerre mondiale constitue un phénomène majeur pour de nombreux pays et représente la véritable fin du "long $\mathrm{XIX}^{\mathrm{e}}$ siècle", en Russie son effet est plus considérable encore. La guerre suit en effet de peu des réformes importantes: introduction pour la première fois d'une assemblée parlementaire ; réformes de Stolypin et tentative pour supprimer la commune paysanne. La Première Guerre mondiale devient ainsi un véritable laboratoire d'idées et de pratiques économiques et sociales. Plusieurs travaux récents avaient déjà attiré l'attention sur ces phénomènes. L'ouvrage en question constitue une synthèse efficace des résultats les plus solides obtenus sur ce sujet. Certes, comme toute publication issue d'un colloque, celle-ci est aussi de niveau inégal. Cependant on ne peut qu'admirer la qualité des articles, très bonne dans l'ensemble, ainsi que des articulations entre les différentes parties. Ce résultat s'explique par les capacités organisationnelles du comité scientifique qui est à l'origine du colloque, tant du côté américain (L. Haimson, W. Rosenberg, R. Zelnik, Z.Galili) que russe (V.Ju. Černjaev, B. I. Kolonickij, S.I. Potolov et surtout N.N.Smirnov, rédacteur en chef et véritable coordinateur de l'ouvrage). En particulier, le choix de reproduire non seulement les articles, mais aussi les débats ayant animé le colloque se révèle particulièrement fécond. Il permet de rendre compte de la richesse des discussions ainsi que de la force heuristique des sujets traités. De nombreuses pistes de recherche surgissent de ces débats.

2 L'ouvrage se compose de six parties principales: théorie, concepts et méthodologie; guerre et société; politique; culture et culture politique; empire et mouvements nationaux ; économie. 
3 La première partie est composée des interventions de Leopold Haimson sur la crise politique et sociale depuis la veille de la Première Guerre mondiale jusqu'à la révolution de Février ; de R. S. Ganelin et M. F. Floronskij sur l'activité du Conseil des ministres en 1915-1916; de D. Orlovsky sur la mémoire de la guerre en Russie et en URSS, et enfin de V. L. D'jakov et L. G. Protasov sur les perceptions de la guerre par la société. L'article de Haimson permet de trancher les nombreuses ambiguïtés de l'historiographie à propos du lien entre réformes tsaristes, guerre et révolution. En particulier, l'auteur conteste la thèse selon laquelle, sans la guerre, la Russie aurait directement évolué vers un système à l'« occidentale ». Au contraire, il met en évidence les limites des réformes et les tensions qui minaient la société russe déjà à la veille de la guerre et que cette dernière n'a fait qu'exacerber. Selon l'auteur, les contraintes auxquelles était soumis le système parlementaire et politique permettent de douter de l'existence d'une véritable société civile en Russie. De même, l'hostilité des paysans envers les réformes de Stolypin témoigne de la spécificité des valeurs agraires russes qui seraient dès lors difficilement réductibles à un modèle simpliste de propriété privée. Ces conclusions peuvent être partagées dans leur ensemble. Deux aspects soulèvent toutefois des doutes. Tout d'abord, la force du mouvement ouvrier et des grèves ouvrières apparaît en partie surestimée afin de rendre compte de l'échec du régime de Février - ceci dans la mesure où la population ouvrière et l'industrie demeuraient plutôt secondaires par rapport au secteur agricole. En outre, l'attitude certes hostile des paysans à l'égard de la privatisation des communes ne saurait faire oublier l'appropriation de ces réformes à l'échelle locale où l'aménagement du territoire acquiert souvent une dimension différente de la «privatisation» et se rapproche plutôt du mouvement coopératif en plein essor à ce moment.

4 Le travail d'Orlovsky est novateur et comble une lacune importante: il analyse la manière dont la mémoire de la Première Guerre mondiale a été construite et a évolué à des époques différentes. Ainsi, à la différence de la plupart des pays européens, après 1917 aucune mémoire officielle de la guerre ne voit le jour en Russie, si ce n'est pour mettre en évidence les prémisses de la révolution d'Octobre. Ce silence relatif, concernant notamment les soldats, a continué jusqu'à nos jours. L'auteur fait également état des tentatives actuelles, en Russie, pour remédier à cette lacune. On regrettera cependant qu'Orlovsky, s'il avance des hypothèses fortes permettant d'expliquer le silence à l'époque soviétique, ne cherche pas à rendre compte de la « redécouverte » actuelle de la Première Guerre mondiale. Il faudrait également mieux qualifier la notion de « mémoire collective » qui, dans le texte, apparaît comme une notion a-historique dans laquelle on retrouve à la fois mémoires de guerre, héros nationaux et tentatives pour donner un nom aux millions de soldats tombés.

5 La deuxième section de l'ouvrage ("Guerre et société ») s'ouvre avec l'article solide et stimulant de Peter Holquist. Cas assez rare dans l'historiographie de la Russie, l'auteur s'efforce de placer un phénomène important en Russie (en l'occurrence la mobilisation) dans un contexte européen. À cette fin, il commence par mettre en évidence les racines de l'économie de guerre et de la mobilisation (la guerre totale) dans les expériences accumulées en Russie et en Europe pendant le $\mathrm{XIX}^{\mathrm{e}}$ siècle. Holquist souligne également l'importance des statistiques en tant qu'instrument à la fois de gestion et de contrôle de la population, notamment du point de vue des ethnies faisant partie de l'empire. Enfin, l'auteur envisage la mobilisation des masses dans la Russie soviétique comme un processus qui, loin d'être imposé par en haut, exprime les bouleversements de la société 
russe pendant la guerre et fait écho aux phénomènes semblables observés dans d'autres pays européens.

6 Ces arguments trouvent un complément indispensable dans l'analyse que fait Ananič des liens entre l'État et la bourgeoisie entrepreneuriale russe. Si cette dernière avait toujours considéré favorablement une intervention, voire un soutien important de l'État à l'activité économique, de leur côté les dirigeants tsaristes adoptent les notions de capitalisme d'État développées en Allemagne depuis Bismarck et renforcées à l'époque de la guerre.

7 Les deux autres interventions consacrées à l'évolution socio-économique mettent en évidence le nouveau rôle des femmes (Peter Gatrell) et l'impact des réfugiés (A. N. Kurcev) dans l'activité économique et dans les hiérarchies sociales. Enfin, V.S. Izmožik détaille le système de contrôle de l'état d'esprit (nastroenie) de la population pendant la guerre et en 1917. L'inspection de la correspondance, non seulement des soldats mais aussi des élites tsaristes, permet de comprendre l'importance de l'héritage légué aux bolcheviks.

8 La troisième section sépare de manière quelque peu artificielle (comme le souligne W. Rosenberg dans la discussion) les aspects proprement politiques des autres. Si V. Ju. Černjaev explique avec finesse les progrès et les limites du processus de démocratisation en Russie, Ju. I. Kir'janov développe un sujet souvent marginalisé dans les ouvrages traitant de l'histoire politique de la Russie, à savoir l'importance et les orientations des partis de droite pendant la guerre.

9 En développant cet argument, la quatrième partie de l'ouvrage fait le point sur la culture, et la culture politique en particulier. L'article de N. N. Smirnov, consacré à l'intelligentsia, est riche d'informations et s'appuie sur une documentation solide. En même temps, comme le souligne Jutta Scherrer dans la discussion, la notion d'intelligentsia utilisée par l'auteur est assez floue; il y inclut en effet intellectuels révolutionnaires, universitaires, élites politiques et administratives tsaristes, spécialistes, etc. Il s'agit là de groupes hétérogènes, tant du point de vue de leur formation et de leur extraction sociale que de leurs orientations politiques. La solution qui consiste à les réunir sous le chapeau commun d'« intelligentsia » semble davantage brouiller qu'éclairer les pistes de recherche.

10 Pour sa part, B. I. Kolonickij propose une intervention tout à fait originale concernant l'image de l'ennemi et l'anglophobie. La finesse avec laquelle l'auteur traite le sujet situe cet article parmi les plus intéressants de l'ouvrage, même si l'on peut peut-être regretter qu'il ne se soit pas employé à montrer les racines, dans la période d'avant 1914, des attitudes russes envers l'Angleterre d'une part, l'Allemagne de l'autre.

11 Également novateur est le travail que Kim Friedlender propose sur les études médicales qu'ont suscitées les chocs d'artillerie subis par les soldats. L'auteur met en évidence le rôle fondateur joué à cet égard par la guerre russo-japonaise. C'est en effet à partir de 1905 que les études de psychiatrie sur les soldats se développent.

12 La cinquième section, consacrée à l'empire et aux mouvements nationaux, permet de faire le point sur un des sujets les plus en pointe ces dernières années. Ainsi, M. von Hagen démontre le rôle de la guerre dans l'émergence de la conscience nationale au sein de plusieurs ethnies de l'empire, et S. M. Išakov étudie l'attitude des musulmans de l'empire envers la guerre. 
13 La sixième et dernière section de l'ouvrage concerne l'économie. Outre l'intervention de T. M. Kitanina sur les rapports russo-germaniques et de S. G. Beljaev sur les pourparlers de Barka à Paris et Londres, on remarquera l'article de Y. Kotsonis sur l'introduction de l'impôt sur le revenu. En s'écartant des études traditionnelles sur ce sujet, l'auteur cherche moins à estimer l'impact économique de cette mesure qu'à mettre en évidence sa signification politique. Par rapport aux autres pays européens qui ont introduit cette mesure à la même époque, le recours à l'impôt sur le revenu est, en Russie, doublement révolutionnaire. Ce type d'impôt est en effet non seulement progressif par rapport à la taxation indirecte (régressive), mais il permet également de dépasser toute séparation de la société en soslovija (états d'Ancien Régime). Cette mesure requiert bien entendu un renouveau profond de l'appareil administratif, censé recueillir et gérer une nouvelle masse d'informations. Nous trouvons sans doute là l'une des meilleures contributions de ce volume et une ouverture vers de nouvelles approches de l'histoire politique et économique de la Russie. Un seul regret, cependant : l'auteur est si désireux de se distinguer des analyses strictement quantitatives et économétriques de la fiscalité qu'il oublie que les conséquences économiques des réformes auraient pu se démontrer autrement. Il paraît en effet difficile d'analyser l'introduction de l'impôt sur le revenu en excluant radicalement son impact sur la dynamique et sur les comportements économiques.

Cet ouvrage portant sur la « Russie de la Première Guerre mondiale », par ses nombreux travaux novateurs, mais aussi par le bilan qu'il fait du travail effectué par l'historiographie russe et anglo-américaine, mérite de devenir un ouvrage de référence pour les études sur la Russie. 